\title{
Post-earthquake building damage assessment in Yushu using airborne SAR imagery*
}

\author{
Dingjian Jin* Xiaoqing Wang Aixia Dou and Yanfang Dong \\ Institute of Earthquake Science, China Earthquake Administration, Beijing 100036, China
}

\begin{abstract}
The synthetic aperture radar (SAR) plays an important role in earthquake emergency response because of its all-time and all-weather imaging capabilities. On April 14, 2010, an $M_{\mathrm{S}} 7.1$ earthquake occurred in Yushu county, Qinghai province of China, causing a lot of buildings collapsed. In this paper, the building damage in Yushu city due to the earthquake was assessed quantitatively using high-resolution X-band airborne SAR image. The features of the buildings with different damage levels (collapsed, partial collapsed, non-collapsed) in the SAR image were analyzed first. Based on these building features, we interpreted the individual building damage in Yushu city block by block and got the numbers of the collapsed, partial collapsed and non-collapsed buildings separately for each block, referring to pre-earthquake QuickBird image when necessary. Let the damage index of individual collapsed, partial collapsed, non-collapsed building be 1, 0.5, 0 respectively, the remote sensing damage index of each block was then calculated through remote sensing damage index equation. Finally, the preliminary quantitative relationship between the remote sensing damage index interpreted from the SAR image and that interpreted from the optical image was built up. It can be concluded that a desirable damage assessment result can be derived from high-resolution airborne SAR imagery.
\end{abstract}

Key words: SAR; remote sensing; Yushu earthquake; damage assessment; seismic damage index; manual interpretation

CLC number: P315.9, TP79 Document code: A

\section{Introduction}

After a destructive earthquake, fast and accurate damage assessment is crucial for effective emergency response. Because of the excellent characteristics such as large area coverage and independence on roads conditions, remote sensing techniques have been applied in earthquake damage monitoring for decades (Wei et al., 2008). With the great advances in remote sensing, earthquake damage information extraction via remote sensing has become practicable in China (Wang et al., 2003). In most cases, the damage assessment were performed with optical remote sensing imagery, such as Landsat data (Yusuf et al., 2001), Terra-ASTER data (Kohiyama and Yamazaki, 2005), Ikonos and QuickBird data (Vu et al., 2005), and airborne optical data (Lei et al., 2010; Guo et al., 2010b). Optical data are easy to inter-

\footnotetext{
* Received 14 August 2011; accepted in revised form 12 September 2011; published 10 October 2011.

+ Corresponding author. e-mail: whujindingjian@163.com

(c) The Seismological Society of China and Springer-Verlag Berlin Heidelberg 2011
}

pret while they cannot be obtained timely on bad weather conditions or at night. Synthetic aperture radar (SAR) remote sensing is relatively insensitive to atmospheric conditions and independent of sun illumination. For this reason, SAR can provide more stable source of remote sensing data, and damage assessment using SAR imagery is very promising.

Among all the damages caused by earthquakes, the building damage contribute mostly to the casualties and property losses, and is the important basis for seismic intensity assessment, therefore building damage assessment using SAR imagery has sparked considerable interest in the remote sensing community. If both the post-earthquake and pre-earthquake SAR data are available, change detection methods can be used to extract the building damage information. The change detection methods utilize mainly the interferometric coherence characteristics (Yonezawa and Takeuchi, 2001; Ito and Hosokawa, 2003; Mansouri et al., 2005), the intensity characteristics (Matsuoka and Yamazaki, 2004, 2005), or the combination of coherence 
characteristics and the intensity characteristics (Arciniegas et al., 2007). The drawback of this kind of method is that the image pairs must have the same acquisition parameters and viewing configuration, a little difference may result in a high rate of false alarms (Brunner et al., 2010). Furthermore, pre-earthquake data are not always available for many regions in the world. With the advent of spaceborne polarimetric SAR systems such as RADARSAT-2, building damage assessment methods using polarimetric SAR data were proposed (Guo et al., 2009, 2010a). Polarimetric SAR data provide more information on damage detection. Unfortunately, only RADARSAT-2 satellite is equipped with commercial full polarimetric SAR at present and the spatial resolution of the polarimetric SAR data is rather low, so it is difficult to identify individual building in such SAR imagery. Besides, GIS layers and optical imagery can provide additional information for SAR imagery. Gamba et al. (2007) proposed a method using SAR imagery in combination with GIS (Geographic Information System) layers for the 2003 Bam earthquake and this method could also get good results for the $2003 \mathrm{Al}-$ geria and 2007 Peru earthquakes (Trianni and Gamba, 2008). The methods using SAR images in combination with optical images were proposed by Chini et al. (2009) for the Bam earthquake and by Brunner et al. (2010) for the 2008 Wenchuan earthquake. These studies showed that multi-source data fusion can improve the precision of the results dramatically.

However, most of the automated building damage assessment methods mentioned above can only provide a coarse results. For quantitative building damage assessment, manual interpretation method is the only choice so far. The spatial resolution of SAR imagery has been improved considerably in recent years. COSMOSkyMed (Covello et al., 2010) and TerraSAR-X (Pitz and Miller, 2010) have a resolution up to $1 \mathrm{~m}$, and airborne SAR systems have a resolution up to decimeter (Zhang et al., 2010). In the high resolution SAR imagery acquired by these sensors, individual building can be identified clearly. Wang et al. (2009a) analyzed the disaster phenomena in high resolution airborne SAR images, including ruined buildings, industrial facilities damage, transportation system damage and landslides. Balz and Liao (2010) detected the building damage in Wenchuan earthquake using post-earthquake COSMOSkyMed and TerraSAR-X images. Their studies focused on the qualitative analysis. However, the ability of SAR imagery to assess the building damage quantitatively has not been extensively investigated.
On April 14, 2010, a $M_{\mathrm{S}} 7.1$ earthquake occurred in Yushu county, Qinghai province of China, causing a lot of buildings collapsed. Remote sensing techniques found a wide application in this earthquake. Shao et al. (2010) calculated the co-seismic deformation of this earthquake using ALOS-PALSAR images. Zhang et al. (2010) and Wang et al. (2010) analyzed the earthquake damages using SAR imagery and multi-source optical imagery, respectively.

In this paper, we will assess the building damage in Yushu city due to the earthquake using high-resolution airborne SAR imagery quantitatively. In section 2, we introduce the remote sensing damage index and its computational model. We describe the remote sensing data used in this study and analyze the features of buildings in SAR image in section 3, and discuss the interpretation result from SAR image and compare it with the result from optical image in section 4 . Finally, we discuss the result and draw conclusions in section 5 .

\section{Remote sensing seismic dam- age index and its computational model}

In order to present seismic damage degree quantitatively, $\mathrm{Hu}$ (1988) proposed the conception of seismic damage index (SDI) after the 1970 Tonghai earthquake. The seismic damage degrees of the buildings vary with different structure types in the same seismic intensity area, thus they can be expressed as SDI which is related to the structure types of the buildings. The average SDI of the building with structure type $i$ in certain survey site is given by

$$
\overline{d_{i}}=\frac{\sum_{j} d_{i j} n_{i j}}{\sum_{j} n_{i j}},
$$

where $d_{i j}$ represents the SDI of the single building with structure type $i$ in damage level $j(j=1,2,3,4,5)$. If the value of SDI equals 1 , it indicates that the building was totally collapsed. If the value of SDI equals 0 , it indicates that the building is intact. Values between 0 and 1 can be divided into several levels; $n_{i j}$ represents the number of buildings with structure type $i$ in damage level $j$.

The seismic performance of buildings with different structure types varies significantly in the same seismic intensity area. Therefore, buildings with different types of structure have different SDI in the same survey site. 
In order to determine seismic damage degree in a certain site, the average SDI of masonry structure $\bar{d}_{b}$ is usually selected as a standard, so the empirical statistics relationship between the average SDI of structure type $i\left(\overline{d_{i}}\right)$ and $\overline{d_{b}}$ is built up. Then the $\overline{d_{i}}$ is converted to the equivalent average SDI $\overline{d_{b i}}$. Thus the composite SDI $D^{\mathrm{G}}$ in this site can be estimated as

$$
D^{\mathrm{G}}=\frac{\sum_{i} \overline{d_{b i}} N_{i}}{\sum_{i} N_{i}},
$$

where $N_{i}$ represents the number of buildings with structure type $i$.

After establishing the composite SDI, the quantitative seismic intensity of this site can be determined according to the relationship between seismic intensity and SDI.

According to the method for determining the SDI with the ground investigation data, Wang et al. (2009b) proposed the conception of remote sensing seismic damage index. Remote sensing seismic damage index $D^{\mathrm{RS}}$ is given by

$$
D^{\mathrm{RS}}=\frac{\sum_{i} \overline{d_{b i}^{\mathrm{RS}}} N_{i}^{\mathrm{RS}}}{\sum_{i} N_{i}^{\mathrm{RS}}},
$$

where $\overline{d_{b i}^{\mathrm{RS}}}$ is the remote sensing seismic damage index of building with structure type $i$, which is computed in equation (1) and converted to the equivalent remote sensing seismic damage index of building with masonry structure; $N_{i}^{\mathrm{RS}}$ is the number of buildings with structure type $i$ interpreted in remote sensing imagery.

There is a slight difference between ground seismic damage index and remote sensing seismic damage index. In ground investigation, the building damage usually has five levels, i.e., the intact, the slightly damaged, the moderately damaged, the seriously damaged and the destruct. However, we can only identify three building damage levels, the collapsed, the partially collapsed and the non-collapsed in remote sensing imagery.

\section{Remote sensing data and build- ing features in airborne SAR imagery}

\subsection{Remote sensing data}

The data used in this study include postearthquake airborne SAR image, post-earthquake airborne optical image and pre-earthquake QuickBird image of Yushu city, see Table 1.
Table 1 Remote sensing data information

\begin{tabular}{ccc}
\hline Data type & Spatial resolution/m & Acquisition date \\
\hline Airborne SAR image & 0.5 & $2010-04-17$ \\
Airborne optical image & 0.2 & $2010-04-17$ \\
QuickBird & 0.6 & $2004-11-06$ \\
\hline
\end{tabular}

The post-earthquake airborne SAR image provided by Chinese National Administration of Surveying, Mapping and Geoinformation was acquired on April 17, 2010. The airborne SAR system operates at X band, and its spatial resolution is $0.5 \mathrm{~m}$ (Zhang et al., 2010). The SAR image is geocoded. The plane flew in the east-west direction, and the buildings were illuminated from the south side.

The post-earthquake airborne optical image was also provided by Chinese National Administration of Surveying, Mapping and Geoinformation, and was acquired at the same time with airborne SAR image. It has three bands and its spatial resolution is $0.2 \mathrm{~m}$. The post-earthquake airborne optical image was used to select the training block in this paper.

The pre-earthquake QuickBird image was provided by Beijing Space Eye Innovation Technology Co., LTD. It was acquired on November 6, 2004 and its spatial resolution is $0.6 \mathrm{~m}$. The QuickBird image was used as a reference image for manual interpretation in this paper.

The post-earthquake SAR and optical images were geometrically corrected to the pre-earthquake optical image using quadratic polynomial method. In addition, the SAR image was despeckled through Lee filter to increase the interpretability of the image.

\subsection{Building features in airborne SAR image}

Due to the side-looking viewing geometry, the buildings have distinctive features in SAR imagery. The images typically consist of four zones: layover area, corner reflection, roof area and shadow area (Balz and Liao, 2010). Figure 1, based on the work by Brunner et al. (2010), shows schematically the reflections from a simple flat-roof building with width $w$, height $h$ and length $l$ viewed by a SAR sensor with incidence angle $\theta$. Section $a$ is the backscatter from the ground; section $b$ is the backscatter from the dihedral corner reflector formed by the building front wall and the surrounding ground; section $c$ indicates the returns from the front wall; section $d$ shows the returns from the building roof; section $e$ represents the shadow area from which no backscattering signal is recorded by the sensor due to the occlusion. The bottom of the figure shows the relative amplitude of backscatter. The backscatter from the dihedral reflec- 
tor $b$ is very strong and exhibits a bright line in SAR imagery. The strength of the double-bounce scattering depends strongly on the height and the aspect angle of the building (Dong et al., 1997). The shadow area shows dark because no backscattering signal is recorded. When $h \leq w \tan \theta$, the case in Figure 1, part of the returns from the building roof $d$, the ground backscatter $a$ and the returns from the front wall $c$ will be overlapped, forming a relatively bright layover area in SAR imagery. While another part of the returns from the building roof will be recorded solely. However, all the returns from the building roof will be recorded before the backscatter from the dihedral reflector when $h>w \tan \theta$, and at this time the layover area is split into an area $a+c+d$ and an area $a+c$. Although the features of the buildings will vary if the building size or the incidence angle changes, the layover area, corner reflection area and shadow area will always exist for an idealized intact building on open ground.

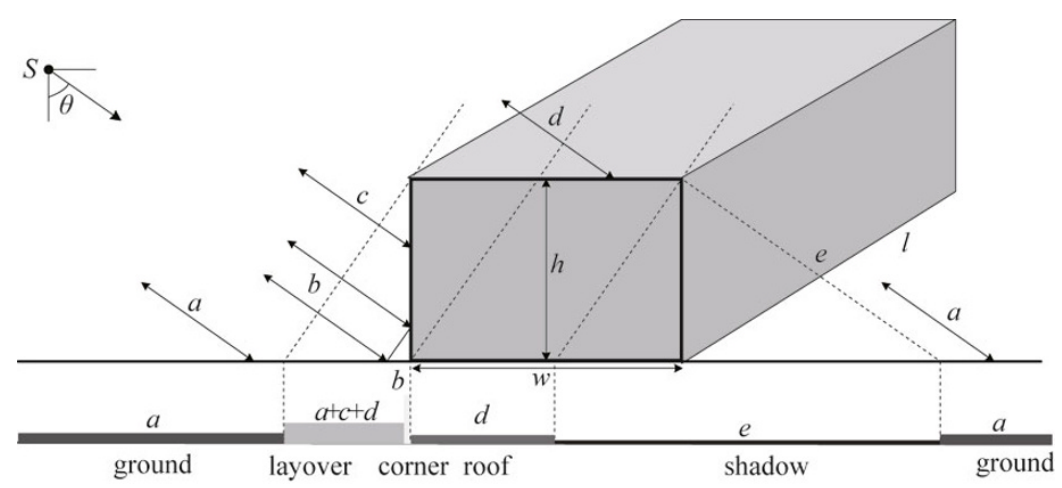

Figure 1 Reflections from a simple flat-roof building (after Brunner et al., 2010).

Figure 2a shows a building in real high resolution airborne SAR image. The same building in the airborne optical image is shown in Figure 2b. The information about the images is described in section 3.1. From the optical image, the building is non-collapsed. In the SAR image, there is a regular and continuous bright line cor- responding to the corner reflection. In front of bright line, there is a layover area. The layover area is disturbed because of the fixtures on the outside of the building. The shadow area behind the bright line is apparent but is hard to distinguish from the background.

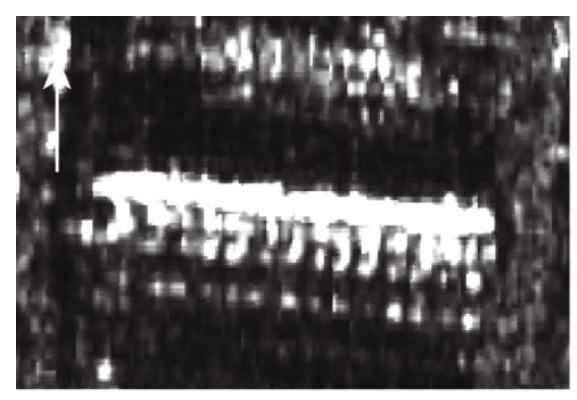

(a)

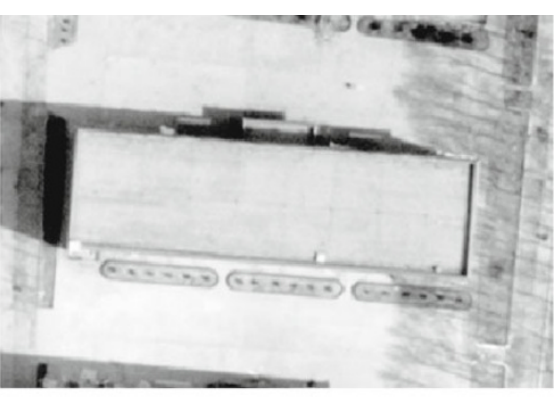

(b)

Figure 2 Example of a non-collapsed building with layover area, bright double-bounce line and shadow area in the SAR image. (a) The airborne SAR image, (b) The airborne optical image. The arrow in (a) indicates the range direction of the image.

However, the shadow area is not always visible. Figure 3 shows two buildings standing close. From the optical image, it can be seen that the two buildings are both non-collapsed. In the SAR image, the backscatter of the two buildings is mixed up and the shadow area of the building in near range can not be seen because it is overlapped by the layover of the building in far range. Fortunately, the regular bright layover area reveals that the building may be non-collapsed. 


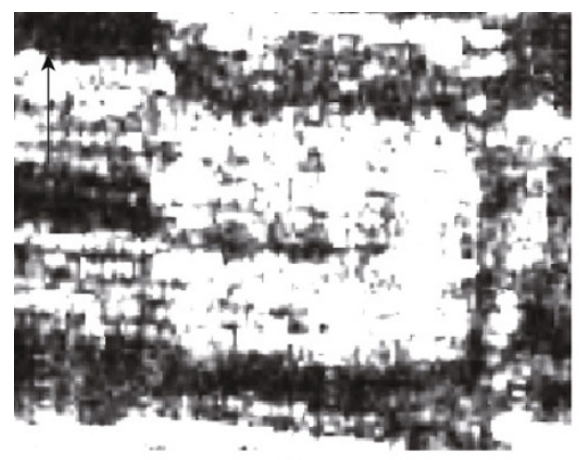

(a)

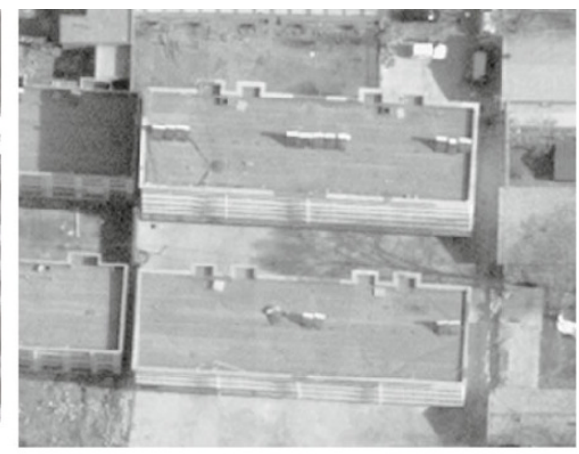

(b)

Figure 3 Example of a non-collapsed building without shadow area in the SAR image. (a) The airborne SAR image, (b) The airborne optical image. The arrow in (a) indicates the range direction of the image.

Generally, once the buildings collapse, the bright layover area and the strong double-bounce line will disappear. An example of collapsed buildings is shown in Figure 4. From the post-earthquake airborne optical image, it can be seen easily that the five buildings are collapsed. In the SAR image, the area corresponding to the buildings looks rough, both the layover and corner reflections on the building edges disappear while the ruins form corner reflectors causing irregular bright spots in the SAR image, the same phenomena were shown by Balz and Liao (2010).

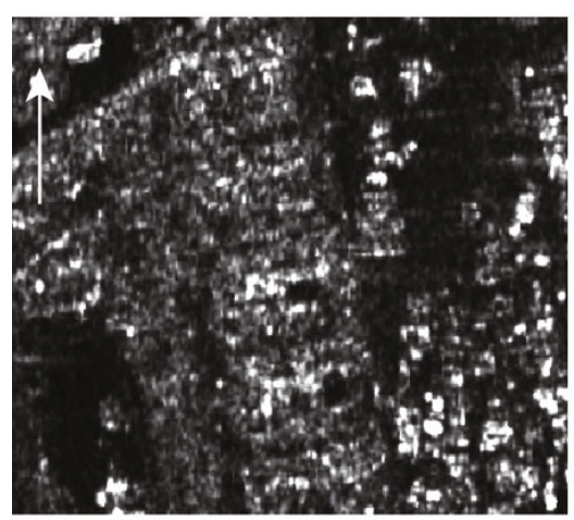

(a)

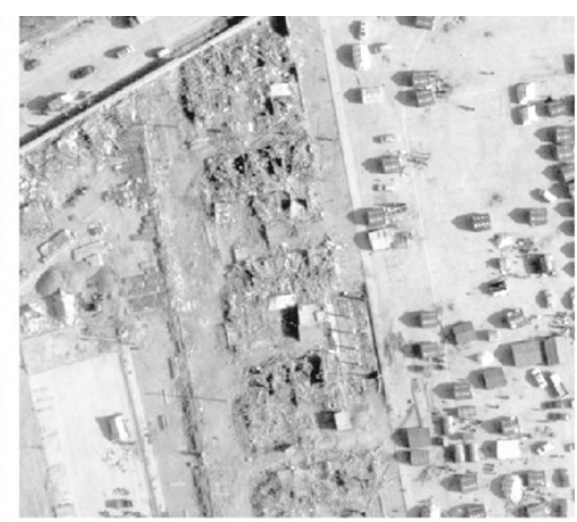

(b)

Figure 4 Example of collapsed buildings. (a) The airborne SAR image, (b) The airborne optical image. The arrow in (a) indicates the range direction of the image.

The above analyses and examples show that the main features of buildings for distinguishing noncollapsed buildings from collapsed buildings are bright double-bounce lines and texture. In SAR imagery, the non-collapsed buildings always have regularly shaped and strong backscattering with trim edges. And in open scene, there may be shadow area accompanied with the strong double-bounce lines. Once the buildings are collapsed, regularly shaped strong backscattering area and the accompanied shadow area both disappear while the ruins may form a corner reflector in a local area and result in irregular bright spots in the SAR imagery.

In some cases, the buildings are not intact but do not collapse completely, we call the buildings are partially collapsed at this time. Figure 5 shows a partially collapsed building. From the optical image, it can be seen that the building is still standing but the middle part of the building is damaged. In the SAR image, there is a bright double-bounce line with a rough section in the middle, which indicates that the middle part of the building may be damaged. 


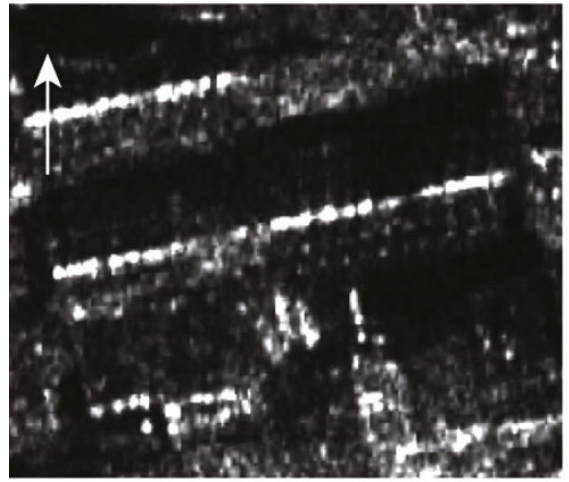

(a)

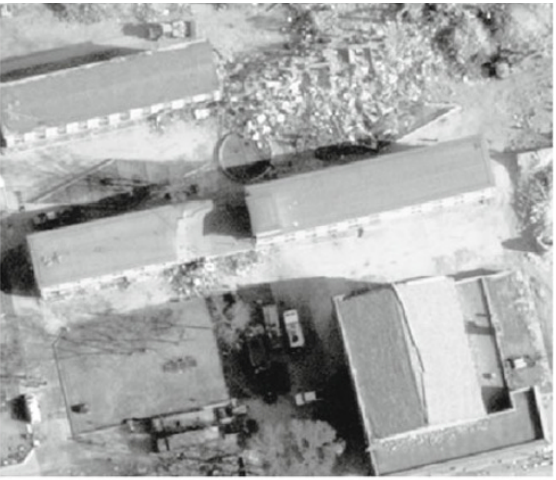

(b)

Figure 5 Example of partially collapsed buildings. (a) The airborne SAR image, (b) The airborne optical image. The arrow in (a) indicates the range direction of the image.

However, building damage assessment using SAR imagery is rather difficult. In real world, there are all kinds of buildings with different structure and size. And the damaged buildings also have different type and extent of destruction. So the features of the collapsed buildings are very complex. Figure 6 shows an example of collapsed building. The post-earthquake airborne optical image shows that the building roof is damaged and the whole building collapsed to north. However, the south wall is still intact. The south wall and the surrounding ground form a dihedral corner reflector though the wall is not vertical. As a result, there is still a bright double-bounce line in the SAR image which indicates wrongly that the building is non-collapsed.

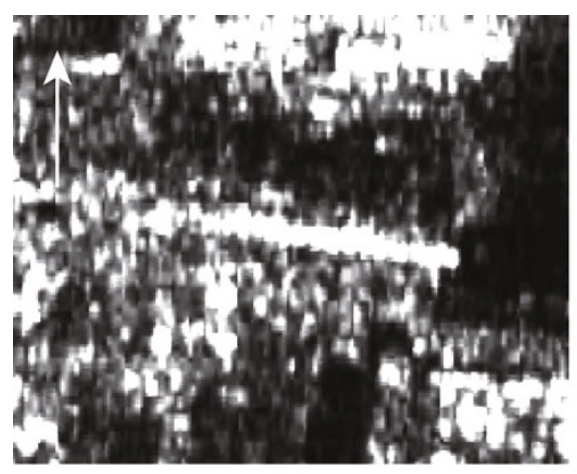

(a)

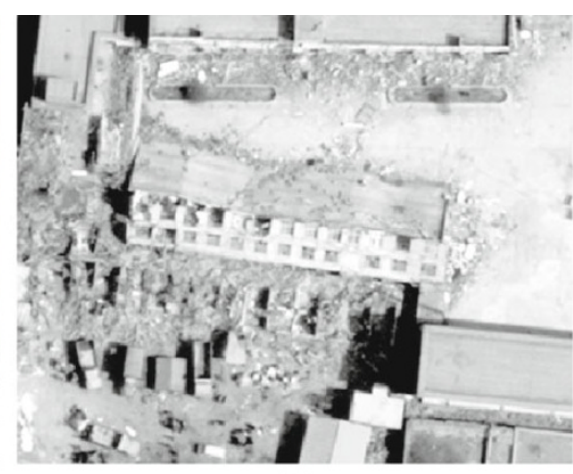

(b)

Figure 6 Example of a collapsed building with bright double-bounce line. (a) The airborne SAR image, (b) The airborne optical image. The arrow in (a) indicates the range direction of the image.

So far, we have only analyzed the characteristics of the flat-roof buildings. It should also be noted that gable-roof buildings have different backscattering characteristics from the flat roof buildings (Bennett and Blacknell, 2003). The SAR image of gable-roof buildings is also characterized by layover area, corner reflector, roof signal and radar shadow (Thiele et al., 2010). Because the gable roof has a slope angle, the backscatter from the roof is mapped to a narrow area and become very strong. The brightest signal appeals if the off-nadir angle equals the slope of the roof. The backscatter from the roof is integrated to a single line at this time. Besides, the dihedral corner reflector formed by the ground and the building wall will cause a strong double-bounce line in the far range. Therefore the parallel line pairs will be observable for the gable roof buildings. Figure 7 shows nine non-collapsed gable roof buildings. The optical image shows that the nine buildings are intact. In the SAR image, the parallel line pairs could clearly be seen, indicating that the buildings are non-collapsed. 


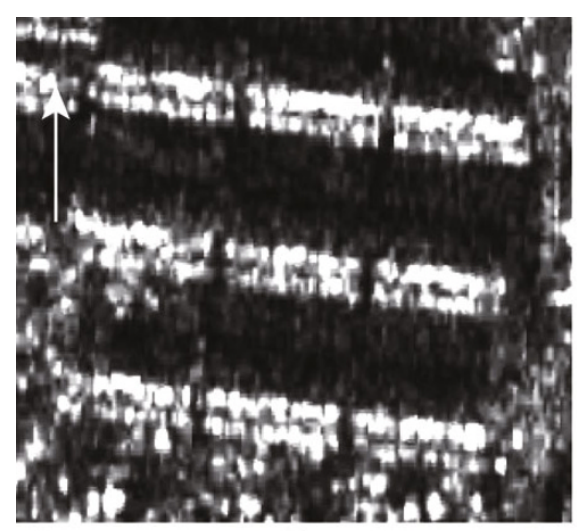

(a)

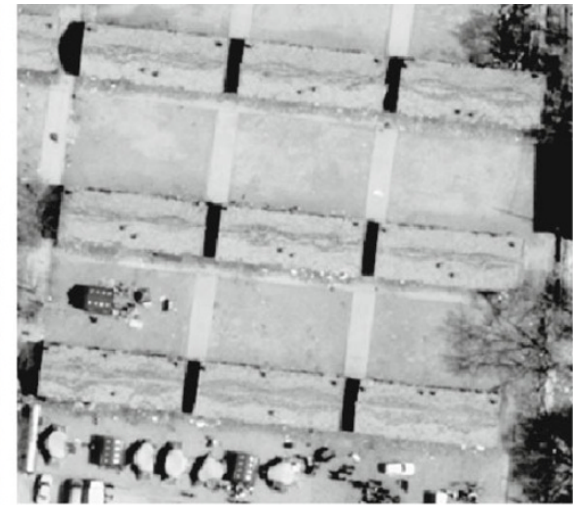

(b)

Figure $7 \quad$ Example of non-collapsed gable roof buildings with parallel line pairs. (a) The airborne SAR image, (b) The airborne optical image. The arrow in (a) indicates the range direction of the image.

\section{Analysis of the remote sensing damage index interpreted from the SAR image}

\subsection{The remote sensing damage index inter- preted from the SAR image}

According to equation (3), the number of buildings with different structures and damage degrees must be obtained in order to calculate the remote sensing damage index. In the airborne SAR image of $0.5 \mathrm{~m}$ resolution, the structure types of buildings are difficult to identify, thus we ignored the structure types and just identified the damage degrees. Let the damage index of individual collapsed, partial collapsed, non-collapsed building be 1, 0.5 and 0 respectively.

Based on these building features, we interpreted the individual building damage in Yushu city block by block and got the numbers of the collapsed, partially collapsed and non-collapsed buildings separately for each block. The results were written into the GIS layer of the city blocks obtained through the pre-earthquake QuickBird image. Building damage interpretation by only post-earthquake SAR imagery is a challenging job, and even become impossible in some cases. However, pre-earthquake high resolution optical imagery which have existed for almost a decade and have already covered large parts of the world can be used to assist the interpretation. In this study, we used the single postearthquake SAR image at most of the time and referred to pre-earthquake QuickBird image only when necessary.

Then we computed the remote sensing damage index for each block according to equation (3), and the result is shown in Figure 8a. The redder the polygon is, the more serious the building damage is. The greener the polygon is, the slighter the building damage is. It can be seen that the building damage in the northeast part of the Yushu city is relatively slighter, while the south and west parts of the city are damaged seriously.

4.2 The quantitative relationship between the remote sensing damage index interpreted from the SAR image and that from the optical image

As discussed above, building damage interpretation from SAR imagery is difficult. It can be imagined that there is a difference between the remote sensing damage index and the ground damage index, and the remote sensing damage index interpreted from airborne SAR imagery can not be used to infer the ground seismic intensity directly. Thus it is necessary to build up the statistical relationship between the remote sensing damage index and the ground damage index which is determined through the practical ground investigation. After that, the remote sensing damage index interpreted from the airborne SAR imagery can be converted to the equivalent ground damage index, and then the seismic intensity in each block can be estimated.

Unfortunately, it is usually hard to investigate each building on ground because of the post-earthquake reconstruction activities. Generally, the remote sensing damage index interpreted from high resolution optical data is more accurate. So we can take the remote sensing damage index interpreted from high resolution optical data as a standard, and build up the relationship between the remote sensing damage index interpreted from airborne SAR data and the remote sensing damage index interpreted from high resolution optical data. And through the statistical relationship between the remote 
sensing damage index interpreted from high resolution optical data and the ground damage index, the relationship between the remote sensing damage index in- terpreted from airborne SAR data and the ground damage index can be built up, so the seismic intensity can be estimated.
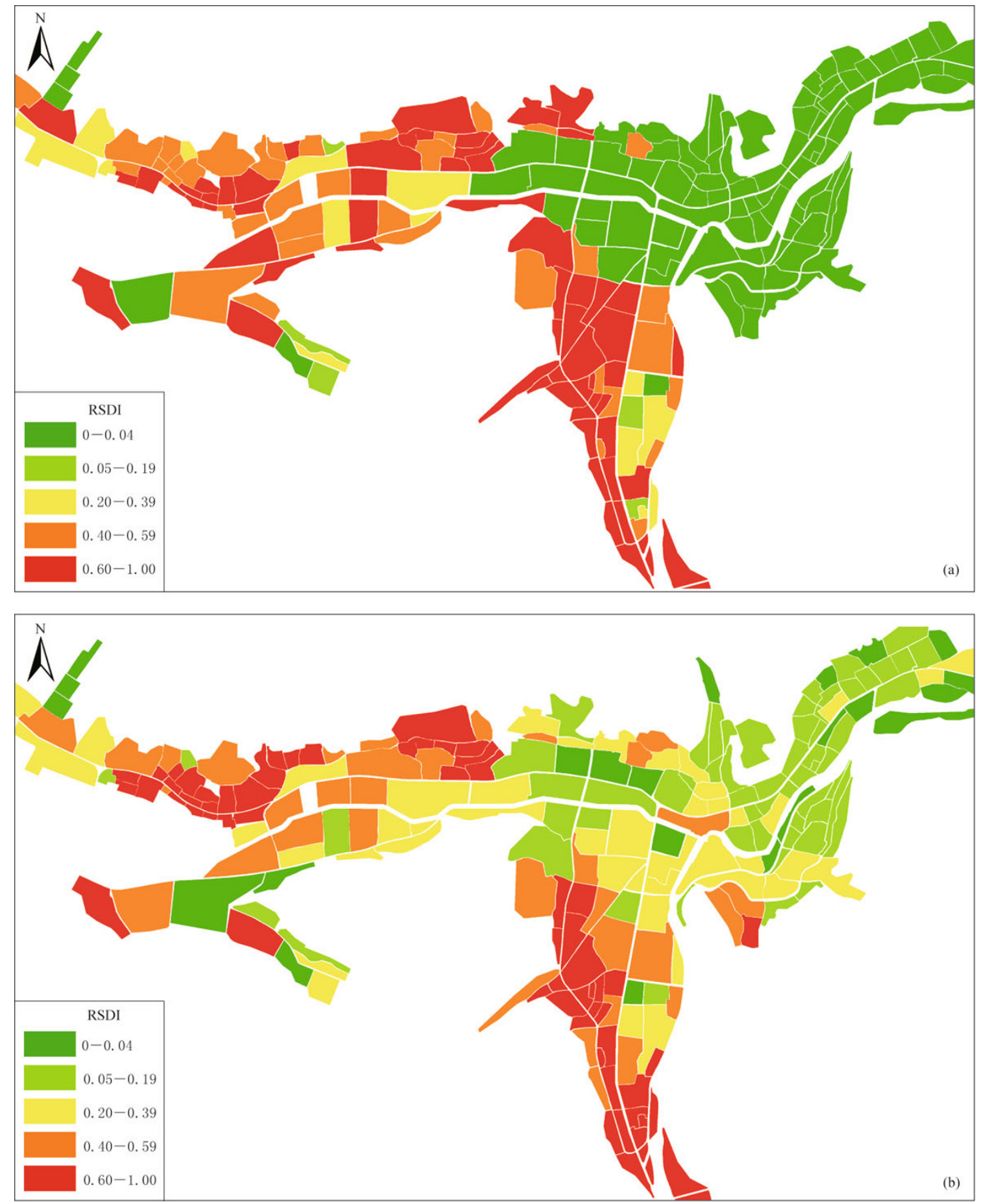

Figure 8 The distribution of the remote sensing damage index (RSDI) interpreted from the airborne SAR image (a) and from the airborne optical image (b) in Yushu city. 
We selected the remote sensing damage index interpreted from the airborne optical image as a reference, shown in Figure 8b, and calculated the quantitative relationship between damage index interpreted from the airborne SAR image and that interpreted from the airborne optical image (Figure 9). Using polynomial fitting method, we obtained the quantitative relationship as follows:

$$
\begin{gathered}
D_{\mathrm{IRSA}}=-0.840 D_{\mathrm{ISAR}}^{2}+1.544 D_{\mathrm{ISAR}}+0.109 \\
\left(R^{2}=0.690\right),
\end{gathered}
$$

where $D_{\text {ISAR }}$ is the damage index interpreted from the airborne SAR image, $D_{\text {IRSA }}$ is the damage index interpreted from the airborne optical image.

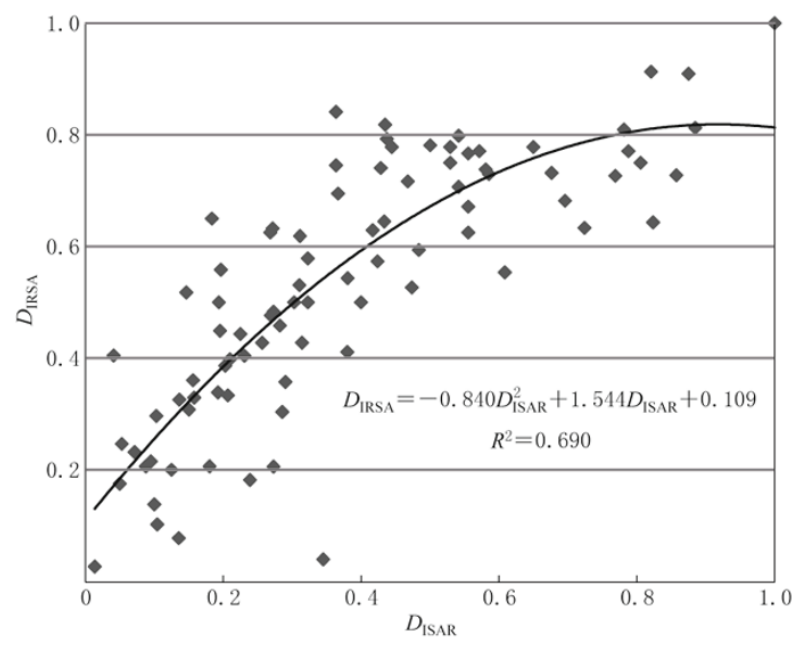

Figure 9 The quantitative relationship between the damage index interpreted from the airborne optical image and that interpreted from airborne SAR image. $D_{\text {ISAR }}$ is the damage index interpreted from the airborne SAR image, $D_{\text {IRSA }}$ is the damage index interpreted from the airborne optical image.

The result shows that there is an obvious statistical relationship between the remote sensing damage index interpreted from airborne SAR image and remote sensing damage index interpreted from airborne optical image, the correlation coefficient is 0.83 . In fact, the interpretation results from two datasets are very close in most blocks.

\section{Discussion and conclusions}

In this paper, we assessed quantitatively the building damage in Yushu city due to the earthquake using high resolution airborne SAR image acquired after this event. We analyzed the building features in high res- olution airborne SAR image firstly. For building damage identification, the double-bounce effects, shape and the shadow are the most important features. Based on these building features, we interpreted manually the individual building damage in Yushu city block by block and got the numbers of the collapsed, partial collapsed and non-collapsed buildings separately for each block, referring to pre-earthquake QuickBird image when necessary. Then the remote sensing seismic damage index was calculated for each block based on the remote sensing seismic damage index computational model which was developed for optical remote sensing and modified slightly for SAR remote sensing. We further built up the quantitative relationship between the damage index interpreted from airborne SAR image and that interpreted from airborne optical image. The result shows that there is an obvious statistical relationship between the remote sensing damage index interpreted from airborne SAR image and remote sensing damage index interpreted from airborne optical image. So it can be concluded that it is possible to get a relatively accurate result for building damage assessment from high resolution SAR imagery.

At the same time, it should be noted that there was still some discreteness in the result interpreted from the SAR image compared to the result interpreted from the optical image. The discreteness is caused by many factors. Firstly, the spatial resolution of the SAR image is lower than that of the optical image. Some irregular or small buildings can be identified in the optical image with a resolution of $0.2 \mathrm{~m}$ while can not be recognized in the SAR image with a resolution of $0.5 \mathrm{~m}$, so the total number of buildings in a block may vary in these two datasets. As a result, the damage index interpreted in these two datasets may vary. Secondly, the imaging geometry of SAR sensor also had an impact. Because of the special imaging geometry of SAR sensors, the neighboring buildings and local topography can strongly influence the appearance of the buildings. In the steep areas or dense built-up areas, the buildings may become even undistinguishable in SAR imagery (Thiele et al., 2007). Thirdly, the pre-earthquake QuickBird image was acquired a long time ago. The city had a lot of changes because of the construction of the city during the time gap between the acquisition of image and the earthquake. These changes may be regarded wrongly as the effect of the earthquake. Furthermore, the interpretation of SAR image and the interpretation of optical image were carried out by different interpreters. The experiences and the skill of the interpreters may affect the 
results to a certain degree.

The quantitative relationship between the damage index interpreted from airborne SAR image and that interpreted from airborne optical image built up in this paper could be used to estimate the seismic intensity in the future. However, as there was still some discreteness, the result was just a preliminary achievement. The quantitative relationship is just a special case in Yushu city for airborne SAR imagery. For different images or different study area, the results may be different. The quantitative relationship should be examined with more earthquake cases and SAR imagery and be modified continuously.

Extracting building damage information from SAR imagery is a challenging job at present. Generally, manual interpretation method can achieve better results than automated approaches. But even if using manual interpretation method, the results can not be as good as that from optical imagery. Besides, the interpreter must be trained before he can interpret the SAR imagery effectively. Despite that, the remarkable capabilities such as weather-and-sunlight independence still make SAR a powerful tool for earthquake emergency response. With more and more high resolution and short repeat time SAR systems become available, the SAR remote sensing will play more and more important role. For a long time in the future, the damage assessment using SAR imagery relies mainly on the manual interpretation. How to extract building damage information automatically using the inherent characteristics such as the shape, texture, shadow, polarization of SAR imagery needs to be studied further.

Acknowledgements The authors would like to thank the anonymous reviewers for their critics and suggestions. The work was supported by the Project "Study on the key techniques of remote sensing applied to earthquake emergency management" funded by Ministry of Science \& Technology of China (No. 2009DFA21610).

\section{References}

Arciniegas G A, Bijker W, Kerle N and Tolpekin V A (2007). Coherence- and amplitude-based analysis of seismogenic damage in Bam, Iran, using ENVISAT ASAR data. IEEE Transactions on Geoscience and Remote Sensing 45(6): 1 571-1 581.

Balz T and Liao M S (2010). Building-damage detection using post-seismic high-resolution SAR satellite data. International Journal of Remote Sensing 31(13): 3 3693391.
Bennett A J and Blacknell D (2003). Infrastructure analysis from high resolution SAR and InSAR imagery. In: Proceedings of the 2nd GRSS/ISPRS Joint Workshop on Data Fusion and Remote sensing over Urban Areas. Berlin, Germany, May 22-23, 2003, 230-235.

Brunner D, Lemoine G and Bruzzone L (2010). Earthquake damage assessment of buildings using VHR optical and SAR imagery. IEEE Transactions on Geoscience and Remote Sensing 48(5): 2 403-2 420.

Chini M, Pierdicca N and Emery W (2009). Exploiting SAR and VHR optical images to quantify damage caused by the 2003 Bam earthquake. IEEE Transactions on Geoscience and Remote Sensing 47(1): 145-152.

Covello F, Battazza F, Coletta A, Lopinto E, Fiorentino C, Pietranera L, Valentini G and Zoffoli S (2010). COSMOSkyMed an existing opportunity for observing the Earth. Journal of Geodynamics 49(Special issue): 171-180.

Dong Y, Forster B and Ticehurst C (1997). Radar backscatter analysis for urban environments. International Journal of Remote Sensing 18(6): 1 351-1 364.

Gamba P, Dell'Acqua F and Trianni G (2007). Rapid damage detection in the Bam area using multitemporal SAR and exploiting ancillary data. IEEE Transactions on Geoscience and Remote Sensing 45(6): 1 582-1 589.

Guo H, Li X and Zhang L (2009). Study of detecting method with advanced airborne and spaceborne synthetic aperture radar data for collapsed urban buildings from the Wenchuan earthquake. Journal of Applied Remote Sensing 3: 031695.

Guo H D, Wang X Y, Li X W, Liu G, Zhang L and Yan S Y (2010a). Yushu earthquake synergic analysis using multimodal SAR datasets. Chinese Science Bulletin 55(31): 3 499-3 503.

Guo H D, Zhang B, Lei L P, Zhang L and Chen Y (2010b). Spatial distribution and inducement of collapsed buildings in Yushu earthquake based on remote sensing analysis. Science China Earth Sciences 53(6): 794-796.

$\mathrm{Hu}$ Y X (1988). Earthquake Engineering. Seismological Press, Beijing, 92-94 (in Chinese).

Ito Y and Hosokawa M (2003). A degree-of-damage estimation model of earthquake damage using interferometric SAR data. Electrical Engineering in Japan 143(3): 4957.

Kohiyama M and Yamazaki F (2005). Damage detection for 2003 Bam, Iran, earthquake using Terra-ASTER satellite imagery. Earthquake Spectra 21(S1): S267-S274.

Lei L P, Liu L Y, Zhang L, Bi J T, Wu Y H, Jiao Q J and Zhang W J (2010). Assessment and analysis of collapsing houses by aerial images in the Wenchuan earthquake. Journal of Remote Sensing 14(2): 333-344.

Mansouri B, Shinozuka M, Huyck C and Houshmand B (2005). Earthquake induced change detection in the 2003 Bam, Iran, earthquake by complex analysis using Envisat ASAR data. Earthquake Spectra 21(S1): S275-S284. 
Matsuoka M and Yamazaki F (2004). Use of satellite SAR intensity imagery for detecting building areas damaged due to earthquakes. Earthquake Spectra 20(3): 975-994.

Matsuoka M and Yamazaki F (2005). Building damage mapping of the 2003 Bam, Iran, earthquake using Envisat/ASAR intensity imagery. Earthquake Spectra 21(S1): S285-S294.

Pitz W and Miller D(2010). The TerraSAR-X satellite. IEEE Transactions on Geoscience and Remote Sensing 48(2): 615-622.

Shao Y, Xie C, Yue Z Q, Wan Z, Wang S A, Bian X L, Gong H Z and Zhang F L (2010). Co-seismic ground deformation of Yushu earthquake detected with D-InSAR technique. Journal of Remote Sensing 14(5): 1 029-1 037.

Thiele A, Cadario E, Schulz K and Soergel U (2010). Analysis of gable-roofed building signature in multiaspect InSAR data. IEEE Geoscience and Remote Sensing Letters 7(1): 83-87.

Thiele A, Cadario E, Schulz K, Thönnessen U and Soergel U (2007). Building recognition from multi-aspect highresolution InSAR data in urban areas. IEEE Transactions on Geoscience and Remote Sensing 45(11): 3 5833593.

Trianni G and Gamba P (2008). Damage detection from SAR imagery: Application to the 2003 Algeria and 2007 Peru earthquakes. International Journal of Navigation and Observation 2008: 1-8.

Vu T T, Matsuoka M and Yamazaki F (2005). Detection and animation of damage using very high-resolution satellite data following the 2003 Bam, Iran, earthquake. Earthquake Spectra 21(S1): S319-S327.

Wang C, Zhang H, Wu F, Zhang B, Tang Y X, Wu H G, Wen X Y and Yan D M (2009a). Disaster phenomena of Wenchuan earthquake in high resolution airborne synthetic aperture radar images. Journal of Applied Remote
Sensing 3: 031690.

Wang L T, Wang S X, Zhou Y, Wei C J, Xu L P and Wang A H (2010). Urgent monitoring and analysis on Yushu earthquake using remote sensing. Journal of Remote Sensing 14(5): 1 053-1 066.

Wang X Q, Wang L, Zhang X H, Ding X, Qiu H, Dong Y F, Dou A X, Zhang F Y and Liu Z T (2009b). Primary quantitative study on earthquake damage extracted from remote sensing imagery - A case study of seismic damage in the urban area of Dujiangyan due to the $M_{\mathrm{S}} 8.0$ Wenchuan earthquake. Earthquake Research in China 23(4): 392-401.

Wang X Q, Wei C J, Miao C G, Zhang J F, Shan X J and Ma Q Z (2003). The extraction of seismic damage from remote sensing images - A case study of BachuJiashi earthquake with $M_{\mathrm{S}}=6.8$ occurred on Feb. 24, 2003. Earth Science Frontiers 10(S1): 285-291 (in Chinese with English abstract).

Wei C J, Liu Y L, Wang S X, Zhang L F and Huang X X (2008). Investigation and assessment of damage in earthquake Wenchuan Sichuan quake based on remote sensing. Journal of Remote Sensing 12(5): 673-682 (in Chinese with English abstract).

Yonezawa C and Takeuchi S (2001). Decorrelation of SAR data by urban damages caused by the 1995 HyogokenNanbu earthquake. International Journal of Remote Sensing 22(8): 1 585-1 600.

Yusuf Y, Matsuoka M and Yamazaki F (2001). Damage assessment after 2001 Gujarat earthquake using Landsat-7 satellite images. Journal of the Indian Society of Remote Sensing 29(1): 233-239.

Zhang J X, Huang G M and Liu J P (2010). SAR remote sensing monitoring of the Yushu earthquake disaster situation and the information service system. Journal of Remote Sensing 14(5): 1 046-1 060. 